\title{
Effect of equation identification status and correlation between error terms on estimators of a system of simultaneous equations model
}

\author{
${ }^{1}$ Kayode Ayinde, ${ }^{2}$ T. L. Johnson and ${ }^{3}$ B.A. Oyejola \\ ${ }^{1}$ Department of Pure and Applied Mathematics, Ladoke Akintola University of Technology, \\ P.M.B.4000, Ogbomoso, Oyo State, Nigeria.Email: bayoayinde@yahoo.com \\ ${ }^{2}$ Department of Statistics, University of Ibadan, Ibadan, Oyo State, Nigeria. \\ ${ }^{3}$ Department of Statistics, University of Ilorin,P.M.B. 1515, Ilorin, Kwara State, Nigeria. \\ email: boyejola@unilorin.edu.ng
}

\begin{abstract}
Violation of the assumption of lack of correlation between the independent variables and error term of a single equation model led to the development of a system of simultaneous equations model whose error terms might be correlated. Moreover in a system of simultaneous equations model, there arises the problem of equation identification. This paper therefore attempts to investigate the effect of these problems on the estimators of the parameter of a system of simultaneous equation model using a Monte Carlo approach. A two equation model with one exactly identified equation and the other over identified was considered. The levels of correlation between error terms $(\lambda)$ were specified as $0.3,0.6$, and 0.9 at three sample sizes $(20,50$ and 100). Six estimation methods; Ordinary Least Squares (OLS), Indirect Least Squares (ILS), Limited Information Maximum Likelihood (LIML), Two Stage Least Squares (2SLS), Full Information Maximum Likelihood (FIML) and Three Stage Least Squares (3SLS); were ranked according to their performances using the finite properties of estimators' criteria which are bias, absolute bias, variance and mean squared error. An estimator is considered best at a specified sample size if it has minimum total rank over the parameters of the equation and the criteria. Results show that the parameter estimation of exactly and over identified equations are best when estimated with either FIML or 3SLS.
\end{abstract}

Keywords: Equation Identification Status, Exactly identified equation, Over Identifiedequation, Correlation between error terms, Estimators.

\section{INTRODUCTION}

When a two-way causation occurs in a function, a single equation model cannot be used to describe the relationships between the relevant variables. A system of simultaneous equations has to be used. In simultaneous equations model, the dependent variable $Y$ and independent variables now appear as endogenous variables as well as explanatory variables in other equation(s) of the model (Koutsoyiannis, 2003). Consequently, the error terms of the equations are inevitably correlated. This has not been directly studied but has been examined unnoticeably through the formation of variancecovariance structure of the error terms of simultaneous equation model (Oduntan, 2004).

Moreover in formulating simultaneous equations, the problem of equation identification arises which eventually becomes a very fundamental prerequisite for model parameter estimation of the system of simultaneous equations model. Johnston (1998) defined this problem as the problem of estimating the parameters of the structure presumed to have generated the data on the endogenous variables from the parameters of the likelihood function. The identification of the parameters of any equation is established if it can be proved that its statistical form is unique. But if its statistical form is not unique that equation is unidentified. A system is thus unidentified if at least one of its equations is not identified. If an equation is identified it is either exactly or over-identified. If an equation is over-identified, it may not be possible to obtain unique structural form parameters from the reduced form parameters. There are two basic rules for identification. They are the order condition and the rank condition. The order condition is a necessary but not sufficient condition whereas the 
Am. J. Sci. Ind. Res., 2011, 2(2): 184-190

rank condition is a sufficient condition. These two rules have been discussed by Koutsoyiannis (2003), Fomby et.al (1988), and many other authors.

Different estimating methods of a system of simultaneous equations model have been given in literature (Brundy and Jorgensen (1971, 1973), Madansky (1976), Fisher (1991), Castineira and Nunes (1999)). These include the Ordinary Least Squares (OLS), Indirect Least Squares (ILS), Two stage Least Squares (2SLS), Limited Information Maximum Likelihood (LIML), Three Stage Least Squares (3SLS) and Full Information Maximum Likelihood (FIML). Castineira and Nunes (1999) classified these methods of estimation into three approaches; the naïve, the limited information and the full information approach. The naïve approach uses the methodology of the OLS estimator as the parameters of each equation are estimated separately. Under certain conditions this method gives the best linear unbiased estimates (BLUE); but if the assumptions are relaxed the estimates are not BLUE. In the Limited Information (LI) approach, estimation of the parameters of the whole system is just like that of the OLS in that parameters of each equation are estimated one at a time. However, unlike OLS it differentiates between explanatory, endogenous and included exogenous variables. Therefore, it uses all identifying restrictions that belong to that equation. It does not require information on the specifications of the other equations in the system, especially the identifying restrictions on them. This class includes ILS, 2SLS and LIML. In the Full Information (FI) approach, the entire system is estimated simultaneously using all the information available on each of the equations of the system. It estimates all the structural parameters given in the model and all the identifying restrictions on each equation of the system. 3SLS and FIML belong to this category. All of these estimators have not only been discussed extensively by many authors including Koutsoyiannis (2003), Castineira and Nunes (1999), Gujarati (2005) and many others but have also been incorporated into some econometric software including the Time Series Process (TSP).

This paper attempts to study the effect of equation identification status and correlation between error terms on estimators of parameters of a system of simultaneous equation model using Monte Carlo approach.

\section{METHODOLOGY}

Consider a two-equation model of the form:

$$
\begin{aligned}
& y_{1 t}=\beta_{12} y_{2 t}+\gamma_{11} x_{1 t}+\gamma_{12} x_{2 t}+u_{1 t} \\
& y_{2 t}=\quad \gamma_{22} x_{2 t}+\gamma_{23} x_{3 t}+u_{2 t}
\end{aligned}
$$

Where $y_{1 t}$ and $y_{2 t}$ are the endogenous variables, $x_{1 t}, x_{2 t}$ and $x_{3 t}$ are exogenous variables, $u_{1 t}$ and $u_{2 t}$ are assumed to be well behaved with a multivariate normal distribution $u \sim N I D(0, \Sigma)$, and $\beta_{12}, \gamma_{12}, \gamma_{22}$ and $\gamma_{23}$ are the structural parameters of the model.

Equation (i) is exactly identified while equation (ii) is over identified by both order and rank condition. The reduced form of the equation is of the form

$$
\begin{aligned}
& y_{1 t}=\pi_{11} x_{1 t}+\pi_{12} x_{2 t}+\pi_{13} x_{3 t}+v_{1 t} \\
& y_{2 t}=\pi_{21} x_{1 t}+\pi_{22} x_{2 t}+\pi_{23} x_{3 t}+v_{2 t}
\end{aligned}
$$

Thus from equation (i) of (1), the reduced form can be obtained by substituting equation (ii) into equation (i). This gives

$$
\begin{aligned}
& y_{1 t}=\beta_{12}\left(\gamma_{22} x_{2 t}+\gamma_{23} x_{3 t}+u_{2 t}\right)+\gamma_{11} x_{1 t}+\gamma_{12} x_{2 t}+u_{1 t} \\
& y_{1 t}=\gamma_{11} x_{1 t}+\left(\beta_{12} \gamma_{22}+\gamma_{12}\right) x_{2 t}+\beta_{12} \gamma_{23} x_{3 t}+\beta_{12} u_{2 t}+u_{1 t}(3)
\end{aligned}
$$

From equations (2) and (3)

$$
\begin{aligned}
& \pi_{11}=\gamma_{11}, \pi_{12}=\beta_{12} \gamma_{22}+\gamma_{12}, \pi_{13}=\beta_{12} \gamma_{23} \text { and } \\
& v_{1 t}=\beta_{12} u_{2 t}+u_{1 t}
\end{aligned}
$$

Comparing equation (ii) of (1) with equation (iv) of (2)

$$
\pi_{21}=0, \pi_{22}=\gamma_{22}, \pi_{23}=\gamma_{23} \text { and } v_{2 t}=u_{2 t}
$$

Therefore,

$$
\begin{aligned}
& \gamma_{11}=\pi_{11} \\
& \gamma_{23}=\pi_{23} \\
& \gamma_{22}=\pi_{22} \\
& \beta_{12}=\frac{\pi_{13}}{\gamma_{23}}=\frac{\pi_{13}}{\pi_{23}}
\end{aligned}
$$


$\gamma_{12}=\pi_{12}-\beta_{12} \gamma_{22}=\pi_{12}-\frac{\pi_{13}}{\pi_{23}} \times \pi_{22}=\pi_{12}-\frac{\pi_{13} \pi_{22}}{\pi_{23}}$

Thus, the formulation of the structural equations in this situation permits the ILS estimator to be used in estimating the parameters since the right hand side of the second equation does not contain an endogenous variable.

For the simulation study, the parameters of the model in equation (1) were fixed as

$\beta_{12}=1.5, \gamma_{11}=0.5, \gamma_{12}=0.8, \gamma_{22}=3.0, \gamma_{23}=2.3$ . The sample sizes (n) were 20 (small), 50 (moderate) and 100 (high). The fixed exogeneous variables were generated such that $X_{i} \sim N(0,1)$ for $i=1,2,3$.The two error terms, $u_{1}$ and $u_{2}$, assumed to be well behaved with a multivariate normal distribution $u \sim N I D(0, \Sigma)$ were also generated to exhibit correlation $\lambda$ using the equations provided and used by Ayinde (2006), Ayinde and Oyejola (2007) and Ayinde (2008). The error terms were thus generated with equation

$$
\begin{aligned}
& u_{1}=\sigma_{1} \sqrt{1-\lambda^{2}} z_{1}+\sigma_{1} \lambda z_{2} \\
& u_{2}=\sigma_{2} z_{2}
\end{aligned}
$$

such that $z_{i} \sim N(0,1)$ for $i=1,2$.

With these specifications, the endogeneous variables were also generated using (1). Equation (ii) of (1) was first generated before (i) of (1). This Monte-Carlo experiment was performed 250 times. For each trial, all the six estimators namely, OLS, ILS, LIML, 2SLS, FIML and 3SLS were used to estimate the parameters of the model. The technique of the ILS method utilized the results of equation (4), (5), (6), (7) and (8). At each scenario, say for instance $n=20$, the estimators were examined and compared using the finite sampling properties of estimators which include bias (B), absolute bias (AB), and variance (Var) and the mean squared error (MSE) criteria. Mathematically, for any estimator $\hat{\beta}$ of $\beta$ in a model

$$
\hat{\hat{\beta}}=\frac{1}{R} \sum_{j=1}^{R} \hat{\beta}_{j}
$$

$$
\begin{gathered}
B(\hat{\beta})=\frac{1}{R} \sum_{j=1}^{R}\left(\hat{\beta}_{j}-\beta\right)=\hat{\beta}-\beta \\
A B(\hat{\beta})=\frac{1}{R} \sum_{j=1}^{R}\left|\hat{\beta}_{j}-\beta\right| \\
\operatorname{Var}(\hat{\beta})=\frac{1}{R} \sum_{j=1}^{R}\left(\hat{\beta}_{j}-\hat{\beta}\right)^{2} \\
\operatorname{MSE}(\hat{\beta})=\frac{1}{R} \sum_{j=1}^{R}\left(\hat{\beta}_{j}-\beta\right)^{2}=\operatorname{Var}(\hat{\beta})+[B(\hat{\beta})]^{2} \\
\text { for } \mathrm{j}=1,2, \ldots, \mathrm{R} .
\end{gathered}
$$

In this study, $\hat{\beta}$ is any of the parameters under consideration in the model of equation (1). For each of the estimators, a computer program was written using Time Series Processor (TSP) software to estimate all the model parameters and to evaluate the criteria. Preferences of estimators were based on bias (closest to zero), minimum absolute bias, minimum variance and minimum mean squared error. Based on each estimate of the parameters, the estimators were ranked in order of their performance at each criterion. The evaluation of methods was done at two levels, the individual criterion and the totality of all the four criteria. For the first level, the ranks based on each criterion were added over the parameters of the model for each method. The overall performances of the estimators were examined by further adding the ranks of the first level over the four criteria. An estimator is considered best if it has minimum total ranks.

\section{RESULTS AND DISCUSSION}

Performances of the estimators on the basis of bias criterion for the exactly identified equation: As shown in Table 1, for the exactly identified equation on the basis of bias criterion, the best estimator is 3SLS. FIML performs either equally well or only slightly different.

LIML and 2SLS are also good estimators particularly when the sample size is moderate $(n=50)$.

For the over identified equation, the best estimator is either FIML or 3SLS. Performance of other estimators are similar when the sample size is small and moderate $(n \leq 50)$. 
Am. J. Sci. Ind. Res., 2011, 2(2): 184-190

Table 1 Summary of the total rank of the parameters based on bias criterion

\begin{tabular}{|c|c|c|c|c|c|c|c|}
\hline \multirow{3}{*}{$\lambda$} & \multirow[b]{3}{*}{ Estimators } & \multicolumn{6}{|c|}{ Equation Identification } \\
\hline & & \multicolumn{3}{|c|}{ Exactly } & \multicolumn{3}{|c|}{ Over } \\
\hline & & $n=20$ & $n=50$ & $n=100$ & $\mathrm{n}=\mathbf{2 0}$ & $n=50$ & $n=100$ \\
\hline \multirow{6}{*}{0.3} & OLS & 13 & 13 & 14 & 9 & 9 & 6 \\
\hline & ILS & 12 & 16 & 9 & 9 & 9 & 12 \\
\hline & LIML & 12 & 7.5 & 13.5 & 9 & 9 & 6 \\
\hline & 2SLS & 12 & 7.5 & 13.5 & 9 & 9 & 6 \\
\hline & FIML & 7 & 11 & 6 & 2.5 & 2 & 7 \\
\hline & $3 S L S$ & 7 & 8 & 7 & 3.5 & 4 & 5 \\
\hline \multirow{6}{*}{0.6} & OLS & 15 & 13 & 13 & 9 & 9 & 5 \\
\hline & ILS & 12 & 14 & 11 & 9 & 9 & 11 \\
\hline & LIML & 10.5 & 8.5 & 9.5 & 9 & 9 & 5 \\
\hline & $2 S L S$ & 10.5 & 8.5 & 9.5 & 9 & 9 & 5 \\
\hline & FIML & 8 & 9 & 8 & 2.5 & 2 & 5 \\
\hline & $3 S L S$ & 7 & 10 & 12 & 3.5 & 4 & 11 \\
\hline \multirow{6}{*}{0.9} & OLS & 15 & 15 & 18 & 9 & 9 & 6 \\
\hline & ILS & 12 & 12 & 15 & 9 & 9 & 12 \\
\hline & LIML & 10.5 & 8.5 & 8.5 & 9 & 9 & 6 \\
\hline & 2SLS & 10.5 & 8.5 & 8.5 & 9 & 9 & 6 \\
\hline & FIML & 9 & 10 & 6 & 3 & 2 & 7 \\
\hline & 3SLS & 6 & 9 & 7 & 3 & 4 & 5 \\
\hline
\end{tabular}

Bold values in the table indicate the lowest ranks indicating the best estimators

Performances of the estimators on the basis of absolute bias criterion: On the basis of absolute bias of the estimators, as show in Table 2, the best estimator is either FIML or 3SLS. However, when the sample size is moderate and correlation between the error terms is not high $(\mathrm{n}=50$, $0.3 \leq \lambda \leq 0.6$ ), the best estimators are LIML and 2SLS even though FIML estimator is almost as good.

For estimating the parameters of the over identified equation, the best estimator is either FIML or 3SLS. The performances of the other estimators are similar.

Performances of the estimators on the basis of variance criterion: As shown in Table 3, the best estimator is either FIML or 3SLS for the exactly identified equation when the variance criterion is used. The performances of LIML and 2SLS estimators are the same.

For the over identified equation, the best estimator is either FIML or 3SLS. The ILS estimator perform better than the OLS, LIML and 2SLS estimators except when the sample size is large $(n=100)$. At this instance, the performances of OLS, LIML and 2SLS are the same.
Performances of the estimators on the basis of mean square error criterion: From Table 4, the best estimator for the exactly identified equation is either FIML or 3SLS except when the sample size is moderate $(n=50)$ and correlation between error terms is low $(\lambda=0.3)$. At this instance, the best estimators are LIML and 2SLS even though the 3SLS is equally good.

Similarly for the over identified equation, the best estimator is either FIML or 3SLS. Although when the sample size is moderate $(n=50)$, the other estimators are equally good. The performances of OLS, LIML and 2SLS are the same. The ILS estimator performs better than the OLS, LIML and 2SLS estimators except when the sample size is large $(n=100)$.

Overall performances of the estimators on the basis of all the criteria for the exactly identified equation: When all criteria are considered together, the best estimators are either FIML or 3SLS for both exactly and over identified equations. The performances of LIML and 2SLS estimators are same. This is shown in Table 5 
Am. J. Sci. Ind. Res., 2011, 2(2): 184-190

Table 2: summary of the total rank of the parameters of the exactly identified equation based on absolute bias criterion

\begin{tabular}{|c|c|c|c|c|c|c|c|}
\hline \multirow{3}{*}{$\Lambda$} & \multirow[b]{3}{*}{ Estimators } & \multicolumn{6}{|c|}{ Equation Identification } \\
\hline & & \multicolumn{3}{|c|}{ Exactly } & \multicolumn{3}{|c|}{ Over } \\
\hline & & $\mathrm{n}=\mathbf{2 0}$ & $\mathrm{n}=\mathbf{5 0}$ & $n=100$ & $n=20$ & $n=50$ & $n=100$ \\
\hline \multirow{6}{*}{0.3} & OLS & 15 & 13 & 15 & 9 & 8.5 & 9 \\
\hline & ILS & 12 & 16 & 14 & 9 & 10.5 & 9 \\
\hline & LIML & 12 & 7.5 & 12.5 & 9 & 8.5 & 9 \\
\hline & $2 S L S$ & 12 & 7.5 & 12.5 & 9 & 8.5 & 9 \\
\hline & FIML & 7 & 11 & 4 & 3.5 & 3 & 3 \\
\hline & $3 S L S$ & 5 & 8 & 5 & 2.5 & 3 & 3 \\
\hline \multirow{6}{*}{0.6} & OLS & 15 & 15 & 15 & 9 & 8.5 & 7 \\
\hline & ILS & 12 & 16 & 14 & 9 & 10.5 & 7 \\
\hline & LIML & 10.5 & 7.5 & 9.5 & 9 & 8.5 & 7 \\
\hline & 2SLS & 10.5 & 7.5 & 9.5 & 9 & 8.5 & 7 \\
\hline & FIML & 7.5 & 9 & 4 & 3 & 3.5 & 2 \\
\hline & $3 S L S$ & 7.5 & 8 & 11 & 3 & 2.5 & 12 \\
\hline \multirow{6}{*}{0.9} & OLS & 15 & 15 & 15 & 9 & 8.5 & 9 \\
\hline & ILS & 16 & 16 & 16 & 9 & 10.5 & 9 \\
\hline & LIML & 11.5 & 11.5 & 11.5 & 9 & 8.5 & 9 \\
\hline & 2SLS & 11.5 & 11.5 & 11.5 & 9 & 8.5 & 9 \\
\hline & FIML & 4 & 6 & 3 & 2.5 & 3.5 & 3 \\
\hline & 3SLS & 5 & 3 & 6 & 3.5 & 2.5 & 3 \\
\hline
\end{tabular}

Bold values in the table indicate the lowest ranks indicating the best estimators

Table 3 summary of the total rank of the parameters of the exactly identified equation based on variance criterion

\begin{tabular}{|c|c|c|c|c|c|c|c|}
\hline \multirow{3}{*}{$\lambda$} & \multirow[b]{3}{*}{ Estimators } & \multicolumn{6}{|c|}{ Equation Identification } \\
\hline & & & Exactly & & & Over & \\
\hline & & $n=20$ & $n=50$ & $n=100$ & $n=20$ & $n=50$ & $n=100$ \\
\hline \multirow{6}{*}{0.3} & OLS & 15 & 15 & 15 & 9 & 9 & 6 \\
\hline & ILS & 16 & 16 & 14 & 7 & 7 & 12 \\
\hline & LIML & 11.5 & 11.5 & 12.5 & 9 & 9 & 6 \\
\hline & 2SLS & 11.5 & 11.5 & 12.5 & 9 & 9 & 6 \\
\hline & FIML & 5.5 & 5.5 & 4 & 4.5 & 3.5 & 5.5 \\
\hline & $3 S L S$ & 3.5 & 3.5 & 5 & 3.5 & 4.5 & 6.5 \\
\hline \multirow{6}{*}{0.6} & OLS & 15 & 15 & 15 & 9 & 9 & 6 \\
\hline & ILS & 14 & 16 & 12 & 7 & 7 & 12 \\
\hline & LIML & 12.5 & 11.5 & 10.5 & 9 & 9 & 6 \\
\hline & 2SLS & 12.5 & 11.5 & 10.5 & 9 & 9 & 6 \\
\hline & FIML & 4 & 6 & 2 & 4.5 & 4.5 & 6 \\
\hline & $3 S L S$ & 5 & 3 & 12 & 3.5 & 3.5 & 6 \\
\hline \multirow{6}{*}{0.9} & OLS & 10 & 15 & 15 & 9 & 7 & 6 \\
\hline & ILS & 15 & 16 & 16 & 7 & 7 & 12 \\
\hline & LIML & 13.5 & 11.5 & 11.5 & 9 & 7 & 6 \\
\hline & 2SLS & 13.5 & 11.5 & 11.5 & 9 & 7 & 6 \\
\hline & FIML & 5 & 5 & 3 & 3.5 & 7 & 6 \\
\hline & $3 S L S$ & 6 & 4 & 6 & 4.5 & 7 & 6 \\
\hline
\end{tabular}

Bold values in the table indicate the lowest ranks indicating the best estimators 
Am. J. Sci. Ind. Res., 2011, 2(2): 184-190

Table 4 summary of the total rank of the parameters of the exactly identified equation based on mean square error criterion

\begin{tabular}{|c|c|c|c|c|c|c|c|}
\hline \multirow{3}{*}{$\lambda$} & \multirow[b]{3}{*}{ Estimators } & \multicolumn{6}{|c|}{ Equation Identification } \\
\hline & & \multicolumn{3}{|c|}{ Exactly } & \multicolumn{3}{|c|}{ Over } \\
\hline & & $\mathrm{n}=\mathbf{2 0}$ & $\mathrm{n}=50$ & $n=100$ & $\mathrm{n}=\mathbf{2 0}$ & $\mathrm{n}=50$ & $n=100$ \\
\hline \multirow{6}{*}{0.3} & OLS & 14 & 13 & 15 & 9 & 7 & 6 \\
\hline & ILS & 12 & 16 & 14 & 7 & 7 & 12 \\
\hline & LIML & 12.5 & 7.5 & 12.5 & 9 & 7 & 6 \\
\hline & $2 S L S$ & 12.5 & 7.5 & 12.5 & 9 & 7 & 6 \\
\hline & FIML & 8 & 10.5 & 4 & 4.5 & 6.5 & 5.5 \\
\hline & $3 S L S$ & 4 & 8.5 & 5 & 3.5 & 7.5 & 6.5 \\
\hline \multirow{6}{*}{0.6} & OLS & 15 & 15 & 15 & 9 & 7 & 6 \\
\hline & ILS & 12 & 14 & 12 & 7 & 7 & 12 \\
\hline & LIML & 12.5 & 8.5 & 10.5 & 9 & 7 & 6 \\
\hline & $2 S L S$ & 12.5 & 8.5 & 10.5 & 9 & 7 & 6 \\
\hline & FIML & 5 & 9 & 4 & 4.5 & 7.5 & 6 \\
\hline & $3 S L S$ & 6 & 8 & 11 & 3.5 & 6.5 & 6 \\
\hline \multirow{6}{*}{0.9} & OLS & 17 & 15 & 15 & 9 & 7 & 6 \\
\hline & ILS & 14 & 16 & 16 & 7 & 7 & 12 \\
\hline & LIML & 11.5 & 10.5 & 11.5 & 9 & 7 & 6 \\
\hline & 2SLS & 11.5 & 10.5 & 11.5 & 9 & 7 & 6 \\
\hline & FIML & 4 & 7 & 3 & 3.5 & 7.5 & 6 \\
\hline & 3SLS & 5 & 4 & 6 & 4.5 & 6.5 & 6 \\
\hline
\end{tabular}

Bold values in the table indicate the lowest ranks indicating the best estimators

Table 5 summary of the total rank of the parameters of the exactly identified equation based on all the criteria

\begin{tabular}{|c|c|c|c|c|c|c|c|}
\hline \multirow{3}{*}{$\lambda$} & \multirow[b]{3}{*}{ Estimators } & \multicolumn{6}{|c|}{ Equation Identification } \\
\hline & & \multicolumn{3}{|c|}{ Exactly } & \multicolumn{3}{|c|}{ Over } \\
\hline & & $\mathrm{n}=\mathbf{2 0}$ & $n=50$ & $n=100$ & $\mathrm{n}=\mathbf{2 0}$ & $n=50$ & $n=100$ \\
\hline \multirow{6}{*}{0.3} & OLS & 57 & 54 & 59 & 36 & 33.5 & 27 \\
\hline & ILS & 52 & 64 & 51 & 32 & 33.5 & 45 \\
\hline & LIML & 48 & 34 & 51 & 36 & 33.5 & 27 \\
\hline & $2 S L S$ & 48 & 34 & 51 & 36 & 33.5 & 27 \\
\hline & FIML & 27.5 & 38 & 18 & 15 & 15 & 21 \\
\hline & 3SLS & 19.5 & 28 & 22 & 13 & 19 & 21 \\
\hline \multirow{6}{*}{0.6} & OLS & 60 & 58 & 58 & 36 & 33.5 & 22 \\
\hline & ILS & 50 & 60 & 49 & 32 & 33.5 & 38 \\
\hline & LIML & 46 & 36 & 40 & 36 & 33.5 & 22 \\
\hline & 2SLS & 46 & 36 & 40 & 36 & 33.5 & 22 \\
\hline & FIML & 24.5 & 33 & 19 & 14.5 & 17.5 & 17 \\
\hline & $3 S L S$ & 25.5 & 29 & 46 & 13.5 & 16.5 & 47 \\
\hline \multirow{6}{*}{0.9} & OLS & 57 & 60 & 63 & 36 & 31.5 & 27 \\
\hline & ILS & 57 & 60 & 63 & 32 & 33.5 & 45 \\
\hline & LIML & 47 & 42 & 43 & 36 & 31.5 & 27 \\
\hline & 2SLS & 47 & 42 & 43 & 36 & 31.5 & 27 \\
\hline & FIML & 22 & 28 & 15 & 12.5 & 20 & 22 \\
\hline & 3SLS & 22 & 20 & 25 & 15.5 & 20 & 20 \\
\hline
\end{tabular}

Bold values in the table indicate the lowest ranks indicating the best estimators 


\section{CONCLUSION}

In the exactly identified equation, the best estimator under bias criterion is 3SLS estimator when the sample size is small $(n=20)$, LIML and 2SLS estimators when the sample size is moderate $(n=50)$, and FIML estimator when the sample size is large $(n=100)$. Under the absolute bias criterion, the best estimator is either FIML or 3SLS or both except when the sample size in moderate $(n=50)$ and correlation between the error terms is low and moderate $(0.3 \leq \lambda \leq 0.6)$; at these instances, the best estimator is LIML and 2SLS. Under the variance criterion, the best estimator is either FIML or 3SLS. Under the mean square criterion, the best estimator is either FIML or 3SLS except when the sample size is moderate $(n=50)$ and correlation between error terms is low $(\lambda=0.3)$; at this instance, the best estimators are LIML and 2SLS. Summarily on the basis of all the criteria, it is observed that the best estimator in estimating the parameters of the model is either FIML or 3SLS or both.

In the over identified equation, it is observed that under each criterion the best estimator is either FIML or 3SLS or both. Summarily based on all the criteria, the best estimator in estimating the parameter of the model is still either FIML or 3SLS or both.

\section{REFERENCES}

Ayinde, K. (2006): A comparative Study of the Performances of the OLS and Some GLS Estimators when Regressors are both stochastic and collinear. West African Journal of Biophysics and Biomathematics. Vol.2: pp.54 - 67

Ayinde, K. (2008): Performances of Some Estimators of Linear Model when Stochastic Regressors are correlated with Autocorrelated Error Terms. European Journal of Scientific Research. 20(3): 558 - 571.

Ayinde, K. and B. A. Oyejola (2007): A comparative Study of the Performances of the OLS and Some GLS
Estimators when Stochastic Regressors are correlated with the error terms. Research Journal of Applied Sciences. 2 (3): $215-220$.

Brundy, J. M. and Jorgenson, D. W. (1971): Efficient Estimation of Simultaneous Equations by Instrumental Variables. Review of Economics and Statistics 53, 1971; pp. $207-224$.

Brundy, J. M. and Jorgenson, D. W. (1973): Consistent and Efficient Estimation of Systems of Simultaneous Equations. P. Zarembka, Ed., Frontiers in Econometrics, Academic Press, 1973.

CASTINEIRA, B. R. and NUNES, L. C. (1999): Testing Endogeneity in a Regression Model; An Application of Instrumental Variable Estimation. Investigation Operative; Vol. 8, Nos. 1, 2 and 3, July - December 1999.

Fisher, F. (1991): Econometrics Essays in Theory and Application. Harvester Wheat Sheaf.

Fomby, B. T., Carter, R.H. and Johnson, R. S. (1988) Advanced Econometric Methods. Springer-Verlag, New York.

Gujarati, D. N. (2005). Basic Econometrics. Fourth Edition. Tata McGraw-Hill Publishing Company Limited.

Johnston, J. (1998): Econometric Methods. $4^{\text {th }}$ Edition. New York: McGraw-Hill.

Koutsoyiannis, A. (2003): Theory of Econometrics; An Introductory Exposition of Econometric Methods. $2^{\text {nd }}$ Edition; Palgrave New York.

Madansky, A. (1976): Foundation of Econometrics. North Holland.

Oduntan, E. A. (2004): A Monte Carlo Study of the Problem of Multicollinearity in a Simultaneous Equation Model. An unpublished Ph.D. Thesis submitted to the Department of Statistics, University of Ibadan, Ibadan, Nigeria.

Theil, H. and A. Zellner (1962): Three Stage Least Squares. Simultaneous Estimation of Simultaneous Equations"; Econometrica, Vol. 30; pp. $54-78$. 